INPLASY

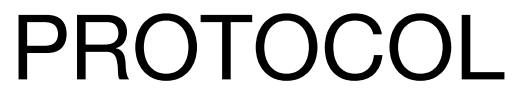

To cite: Vakali et al. The effects of alpha-lipoic acid supplementation on diabetic nephropathy in humans. Inplasy protocol 202060095. doi:

10.37766/inplasy2020.6.0095

Received: 25 June 2020

Published: 25 June 2020

Corresponding author:

Petros Dinas

petros.cd@gmail.com

Author Affiliation:

FAME Laboratory, University of

Thessaly, Greece.

Support: No funding.

Review Stage at time of this submission: Preliminary searches.

Conflicts of interest: No conflict of interest exist.

\section{The effects of alpha-lipoic acid supplementation on diabetic nephropathy in humans}

\author{
Vakali, E1, Rigopoulos, D², Carrillo, A33, Flouris, A4, Dinas, P5.
}

Review question / Objective: To examine the effects of alphalipoic acid supplementation on diabetic nephropathy in humans.

Condition being studied: We are going to explore whether patients with diabetic nephropathy can improve their diabetic nephropathy biological indices via supplementation of alphalipoic acid.

Information sources: Two review team members will independently screen the titles and abstracts of the retrieved publications to select the eligible publications. A third review team member will act as a referee in case of a disagreement between the review team members. We will also ensure that any retracted publications are identified and excluded from the selection outcome. Furthermore, we will locate the full texts that will not be immediately accessible, via emails to the lead authors and/ journals of publication. A full list of the excluded publications will be provided in the final version of the systematic review.

INPLASY registration number: This protocol was registered with the International Platform of Registered Systematic Review and Meta-Analysis Protocols (INPLASY) on 25 June 2020 and was last updated on 25 June 2020 (registration number INPLASY202060095).

\title{
INTRODUCTION
}

Review question / Objective: To examine the effects of alpha-lipoic acid supplementation on diabetic nephropathy in humans.
Rationale: Diabetic nephropathy is characterized by glomerular hypertrophy, renal fibrosis decreased glomerular filtration and proteinuria. It is estimated that the risk for mortality due to kidney 
disease among diabetic patients is $31 \%$, while individuals with diabetes will rise to $>430$ million globally by 2030 . Therefore, interventions towards diabetic nephropathy treatment are heavily warranted. Alphalipoic acid is a natural substance essential for several enzymes that are involved in oxidative metabolism of mitochondria.

Condition being studied: We are going to explore whether patients with diabetic nephropathy can improve their diabetic nephropathy biological indices via supplementation of alpha-lipoic acid.

\section{METHODS}

Search strategy: Two independent investigators performed an algorithmic searching on PubMed central, EMBASE, Web of Science and Cochrane library (trials) databases. These search algorithms were "translated" from one database to another, so that they were recognizable by the corresponding website search engine. A third investigator acted as a referee in disagreements between the two searches. The reference lists of the eligible publications, will also be screened for identifying any eligible to the research question publications, which did not appear in the initial searching.

Participant or population: Diabetic patients suffer from diabetic nephropathy of both sexes, at any age and stage of disease. No restriction on body weight and diet/ physical activity habbits will be applied.

Intervention: Supplementation of alphalipoic acid and/or supplementation of alpha-lipoic acid along with additional interventions (i.e. another supplement, exercise).

Comparator: Pre and post measurements of a single group that recieved an alphalipoic acid intervention or an experimental group and a group that did not receive alpha-lipoic acid and act as controls.

Study designs to be included: Any methodological design.
Eligibility criteria: Inclusion criteria for eligible studies 1. Peer-reviewed experimental/epidemiological studies of any methodological design that examined the effects of alpha-lipoic acid supplementation on diabetic nephropathy in humans. 2. Outputs in any language. 3. No date limits will be applied in the selection of eligible publications. Exclusion criteria for eligible studies 1. Publications that did not examine the effects of alphalipoic acid supplementation on diabetic nephropathy in humans 2 . Studies conducted on animals 3 . Review papers, letters to editors and opinion papers 4 . Theses and dissertations 5. Grey literature (i.e. non-peer reviewed publications).

Information sources: Two review team members will independently screen the titles and abstracts of the retrieved publications to select the eligible publications. A third review team member will act as a referee in case of a disagreement between the review team members. We will also ensure that any retracted publications are identified and excluded from the selection outcome. Furthermore, we will locate the full texts that will not be immediately accessible, via emails to the lead authors and/ journals of publication. A full list of the excluded publications will be provided in the final version of the systematic review.

Main outcome(s): Main outcomes will include any biological index assessed to determine diabetic nephropathy in relation to alpha-lipoic acid supplementation.

Data management: Two review team members will independently extract data from the eligible publications in an appropriate table. In case of disagreement a referee investigator will make an ultimate decision regarding the data that should be extracted. A priori pilot data extraction will be used, to ensure a comprehensive data extraction process. In case that data are missing from a paper we will contact via email the corresponding authors in order to retrieve them. The data that will be included in the final data extraction table are: 1. First author name and year of 
publication 2. Methodological design 3. Population characteristics 4 . Intervention, measurements 5 . Main outcome.

Quality assessment / Risk of bias analysis: The risk of bias assessment will be performed by two independent assessors, while a third investigator will act as a referee. According to the methodological design of each eligible study, we will use three main tools for the risk of bias assessment: a) the Cochrane library tool for assessing the risk of bias in randomised controlled trials, b) the ROBINS-I tool for assessing the risk of bias in studies of nonRCT nature that used an intervention, and c) the RTI Item Bank tool, for assessing the risk of bias in observational studies. The results of the risk of bias assessment will be extracted in relevant tables and figures.

Strategy of data synthesis: For the eligible studies that will not deliver suitable data for a meta-analysis, a summarized qualitative description of their results will be provided. For the eligible studies that will provide available and/or suitable data for a metaanalysis, a random or a fixed-effect metaanalysis model will be used to account for heterogeneity due to differences in study populations, type of infection, interventions, study duration, and other factors. All meta-analyses will be conducted using the RevMan 5.3 software.

Subgroup analysis: Subgroup analysis will include analyses of different categories for age, body mass index, sex, disease stage of diabetes, disease stage of diabetic nephropathy, alpha-lipoic acid supplementation with or without another intervention and different medication.

Sensibility analysis: In case that the data of the eligible publications are suitable, we will assess the applicability of the findings of the systematic review via the Grading of Recommendations Assessment, Development and Evaluation (GRADE) analysis.

Country(ies) involved: Greece, USA.
Keywords: diabetes, nephropathy, alphalipoic acid.

Contributions of each author:

Author 1 - Eleni Vakali.

Author 2 - Dimitrios Rigopoulos.

Author 3 - Andres Carrillo.

Author 4 - Andreas Flouris.

Author 5 - Petros Dinas. 